\title{
Wrinkles: From the Sea to Mathematics
}

\author{
François Laudenbach (Université de Nantes, France)
}

\section{Wrinkles and sealing}

The mathematical meaning of wrinkle and its real-life meaning are very similar, particularly when the word is used to describe features on the surface of the sea. Luckily, I was educated in sailing before learning advanced mathematics so I spent a lot of time looking at wrinkles on the sea. After a long, windless period, it is delightful to observe their birth and the forms they take. Long after learning to sail, I read that these forms bear great similarity to those predicted by R. Thom's singularity theory (see [15]). On one occasion, wrinkles appeared and grew so abruptly that they clearly announced a strong event; at the same time, the on-board radio issued gale warnings for the forthcoming night. Soon the fog cleared, allowing us to see an enormous cumulonimbus cloud, with its white anvil thousands of metres high, indicating the presence of fantastic thermodynamic machinery. Fortunately, this experience concluded safely.

\section{Basics about wrinkles}

Mathematical wrinkles are models for maps $\mathbb{R}^{n} \rightarrow \mathbb{R}^{q}, n \geq q$, restricted to some explicit compact subset. The very primitive wrinkle is the one-variable smooth function whose graph is shown in Figure 1. That function $f$, defined on $[a, b]$, has one maximum and one minimum, both non-degenerate, meaning that the second derivative does not vanish at these critical points; one says that $f$ is a Morse function. It is unique up to reparametrisation of the domain and the range, which can alter, for example, the distance between critical points and critical values.

As $f(a)>f(b)$, the maximum principle for real continuous functions makes it impossible to deform $f$ to a function without critical points if we insist on keeping $f$ unchanged near the boundary points $a$ and $b$. Indeed, there must exist a maximum value greater than $f(a)$ and a minimum value less than $f(b)$.

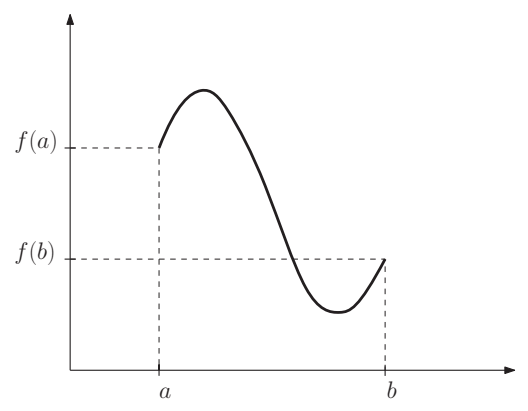

Figure 1. The primitive wrinkle
Nevertheless, the differential $d f$ can be deformed to a non-vanishing differential 1-form, denoted $\mathcal{R} d f$, which coincides with $d f$ near $a$ and $b$. Of course, the corresponding change in $f$ would have to change the value at one endpoint, say $b$. This $\mathcal{R} d f$ is called the regularized differential of $f$. It is unique up to deformation among the non-vanishing differential forms since the constraints near the endpoints define a convex set. The name and notation are due to Y. Eliashberg and N. Mishachev [2], the inventors of mathematical wrinkles.

\section{Wrinkles and immersions}

Historically, this first wrinkle (without its name) appeared soon after S. Smale's breakthrough of the sphere eversion [14], a counter-intuitive phenomenon where it is possible to turn a sphere inside out by allowing only self-intersections but no pinching, that is, by moving the sphere through immersions. The first person who figured out Smale's result was A. Shapiro $(1961)^{1}$ (see, in chronological order, [11], [6] and [9]). The eversion [11], of which Figure 2 shows one stage, exhibits a lot of wrinkles.

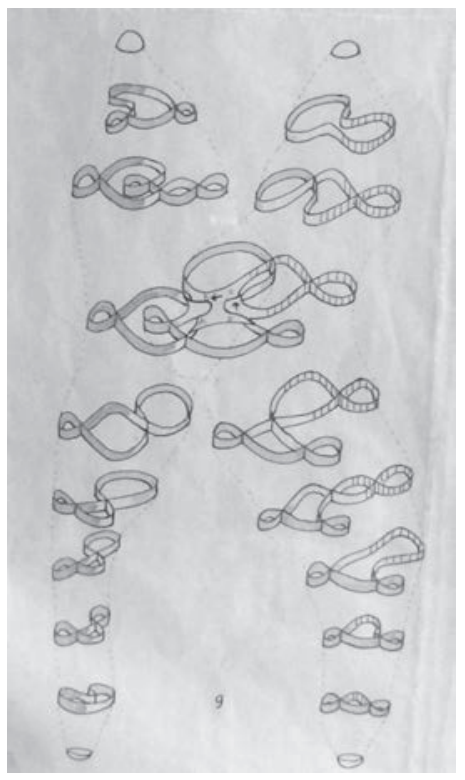

Figure 2. Original drawing for a sphere eversion (courtesy of Anthony Phillips)

1 Quoting the beginning of Francis \& Morin's article: "We dedicate this article to the memory of Arnold Shapiro, who gave the first example of how to turn the sphere inside out, but never published it." 


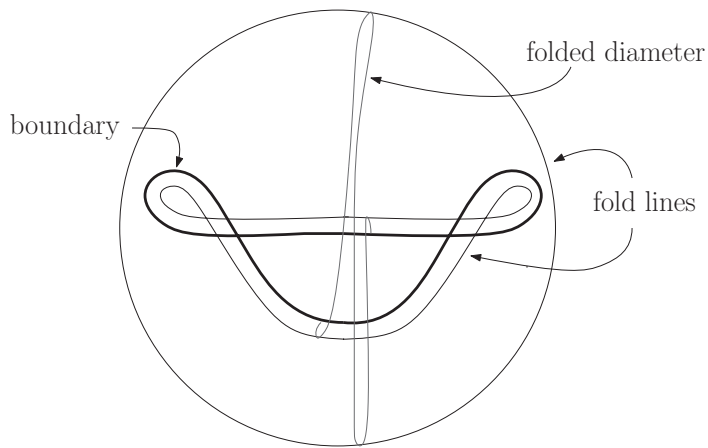

Figure 3. Folded immersion of the 2-disc and one folded diameter

A natural question to ask is which immersions of the $(n-1)$-sphere into $\mathbb{R}^{n}$ extend to an immersion of the $n$-ball. Apart from some obvious homotopy theoretical obstructions, the problem remains open for $n>2$. A complete (but difficult) answer in dimension 2 has been given by S. Blank (see [12]).

In 1966, V. Poenaru [13] showed that relaxing the immersion condition on the $n$-ball by allowing folds (such as, in local coordinates, $x \mapsto x^{2}$ in dimension one or $(x, y) \mapsto\left(x^{2}, y\right)$ in dimension two) made the problem of extension easily solvable. For instance, focusing on $n=2$ to make drawings possible, every immersion $S^{1} \rightarrow \mathbb{R}^{2}$ extends to a folded immersion of the disc to the plane as in Figure 3. In this example, without any fold line, there is no immersion of the disc extending the given immersion. Indeed, the immersion of the disc if it exists must enter the unbounded component of the complement of the given immersed boundary line. But the disc is compact and any point of its image lying at a maximal distance from the origin should be a critical point of the map, which is therefore not an immersion.

This result translates a sort of flexibility in a sense precisely defined by M. Gromov in his seminal book [8]. The fold lines that Poenaru introduces lie parallel to the boundary in a collar neighbourhood in the source disc. Thus, a pair of consecutive such fold lines may be thought of as a oneparameter family of primitive wrinkles. In the example shown in Figure 3, this family is made of folded diameters; one of the diameters is shown.

\section{Wrinkles and the $h$-principle}

The efficiency of what have been called primitive wrinkles above - mostly used in families - is already remarkable when applied to immersions (see W. Thurston's corrugations [16]). Of course, if one aims to apply wrinkles to very general classes of smooth mappings of manifolds, a more elaborate model of wrinkles has to be used. Fortunately, this model, which is global in essence, still involves the two local stable singularities of mappings from plane to plane only, namely the fold and the so-called cusp.

If the source and target are two-dimensional, a wrinkle $W$ consists, as in Figure 4, of a neighbourhood of a disc $D$ in the source, fibred in intervals over an interval $I$, with two fibres tangent to the boundary $\partial D$ at points $c_{1}$ and $c_{2}$, and a smooth map $w: W \rightarrow \mathbb{R}^{2}$. This map is fibred over an interval; its singular locus consists of two cusps in $c_{1}$ and $c_{2}$ and of

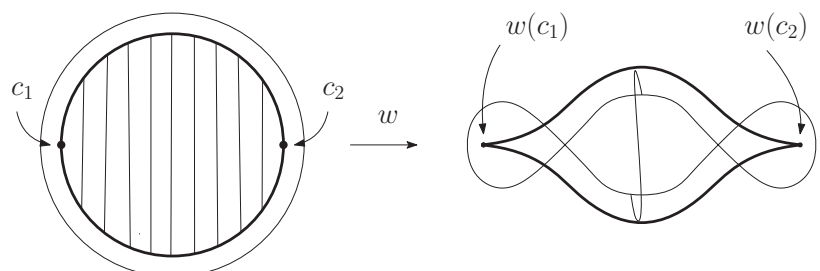

Figure 4. The lips wrinkle has cusps at $c_{1}$ and $c_{2}$

two fold lines, namely the two open $\operatorname{arcs} \partial D \backslash\left\{c_{1}, c_{2}\right\}$ (see Figure 4).

The image of the singular locus looks like lips, a famous figure in Cerf's analysis of pseudo-isotopies [1]. Using horizontal coordinate $y$ in $[-1,1]$ and $x$ in the fibre over $y$,

$$
w(y, x)=\left(y, f_{y}(x)\right),
$$

where $\left(f_{y}(x)\right)_{y}$ is a 1-parameter family of primitive wrinkles from birth to death as $y$ traverses the interval $\left[y\left(c_{1}\right), y\left(c_{2}\right)\right]$. For further extension to high dimension, one requires the symmetry $f_{y}=f_{-y}$. By regularising the differential $d f_{y}$ smoothly in $y$, we get a regularised differential:

$$
\mathcal{R} d w: T_{(y, x)} W \rightarrow T_{w(y, x)} \mathbb{R}^{2} .
$$

The word regularisation translates the fact that the rank of $\mathcal{R} d w$ is maximal on all fibres of the tangent space $T W$. This model extends to a wrinkle $w$ with source and target of dimension $q$, simply by taking $y \in \mathbb{R}^{q-1}$ and rotating the 2dimensional model about the axis $y=0$. Then,

$$
w(y, x)=\left(y, f_{|y|}(x)\right)
$$

and the regularised differential still exists. Finally, one can obtain a non-equidimensional model by enlarging the source with an $(n-q)$-ball (with coordinate $z$ ) and taking the map

$$
w(y, x, z)=\left(y, f_{|y|}(x)+Q(z)\right),
$$

where $Q$ is a non-degenerate quadratic form.

With these models at hand, a wrinkled map $g: M^{n} \rightarrow$ $N^{q}, n \geq q$, between (possibly closed) manifolds is a smooth map that coincides with wrinkle models in finitely many disjoint balls in $M^{n}$ and has maximal rank elsewhere. Eliashberg \& Mishachev [2] state the flexibility of wrinkled maps, in that an $h$-principle in the sense of Gromov holds true for them. To make this more precise, denote by $T M$ and $T N$ the respective tangent spaces and consider the set W(M,N), formed by the collection of wrinkled maps and completed by maps with so-called embryos or unborn wrinkles. This set embeds into the space of bundle epimorphisms $\operatorname{Epi}(T M, T N)$ by the regularised differential operator $\mathcal{R} d$ and this embedding is a homotopy equivalence.

\section{Applications and novelties}

A spectacular application to pseudo-isotopy theory [3] is a strong generalisation of a theorem by K. Igusa (1984) [7], with no restriction on dimension. Namely, any family of smooth functions $\left\{f_{u}: M \times([0,1], 0,1) \rightarrow([0,1], 0,1)\right\}_{u \in S^{k}}$ contracts in the space of Morse functions with the same boundary condition, completed by the embryos (functions with one cubic singularity). 
More recently, Eliashberg \& Mishachev with S. Galatius [5] have shown that wrinkles apply in an area usually reserved to homotopy theorists. The question is to compute the $s t a b l e^{2}$ homology of the mapping class group of a Riemann surface.

Last but not least, thanks to the flexibility of a slightly different object called a wrinkled embedding [4], we witnessed an exceptional event: E. Murphy's breakthrough in high-dimensional contact topology [10]. Thanks to wrinkling techniques (large zig-zags in front projections ${ }^{3}$ ), she discovered the loose Legendrian embeddings into contact manifolds. Such an embedding makes the ambient contact structure flexible. The wrinkle story is clearly far from over.

\section{Acknowledgement}

I am very indebted to Frank Morgan who gave me many suggestions for writing in a simpler language for addressing a large audience. I also feel very grateful to Allyn Jackson for her help in some delicate situations. I express my thanks to Anthony Phillips who offered me a copy of one of his beautiful drawings and to Vincent Borrelli for his careful reading of this note.

\section{References}

[1] Cerf J., La stratification naturelle des espaces de fonctions différentiables réelles et le théorème de la pseudo-isotopie, Inst. Hautes Études Sci. Publ. Math. 39 (1970), 5-173.

[2] Eliashberg Y. \& Mishachev N., Wrinkling of smooth mappings and its applications I, Invent. Math. 130 (1997), 345-369.

[3] _ Wrinkling of smooth mappings-II Wrinkling of embeddings and K. Igusa's theorem, Topology 39 (2000), 711-732.

[4] _ Wrinkled embeddings, Contemporary Mathematics 498 (2009), 207-232; http://dx.doi.org/10.1090/conm/498/09753.

2 Quoted from [5, p. 412]: "stabilization with respect to the genus".

3 Near a Legendrian submanifold, the local model of a contact structure is given by the one-jet space $J^{1}\left(\mathbb{R}^{n}, \mathbb{R}\right)$ equipped with the $2 n$-plane field whose equation reads $d z-\sum p_{i} d q^{i}=0$ in canonical coordinates. The front projection is the projection onto the 0 -jet space $(q, p, z) \mapsto(q, z)$.
[5] Eliashberg Y., Galatius S. \& Mishachev N., Madsen-Weiss for geometrically minded topologists, Geometry \& Topology, 15 (2011), 411-472.

[6] Francis G. \& Morin B., Arnold Shapiro's eversion of the sphere, Math. Intelligencer 2 (1979), 200-203.

[7] Igusa K., Higher singularities are unnecessary, Annals of Math. 119 (1984), 1-58.

[8] Gromov M., Partial Differential Relations, Springer, 1986.

[9] Levy S., Making waves, A guide to the ideas behind Outside In, (text and video), AK Peters, Wellesley (MA), 1995.

[10] Murphy E., Loose Legendrian embeddings in high dimensional contact manifolds, arXiv: 1201.2245.

[11] Phillips A., Turning a surface inside out, Scientific American 214 (May 1966), 112-120.

[12] Poenaru V., Extension des immersions en codimension 1 (d'après S. Blank), exposé $\mathrm{n}^{\circ} 342$, p. 473-505 in: Séminaire N. Bourbaki, vol. 10, 1966-1968, Soc. Math. de France, 1995; https://eudml.org/doc/109745.

[13] Poenaru V., On regular homotopy in codimension 1, Ann. of Math. 83 (2) (1966), 257-265.

[14] Smale S., A classification of immersions of the two-sphere, Trans. Amer. Math. Soc. 90 (1958), 281-290.

[15] Thom R., Structural Stability and Morphogenesis, AddisonWesley, 2nd ed., 1994.

[16] Thurston W., Making waves: The theory of corrugations, in: [9].

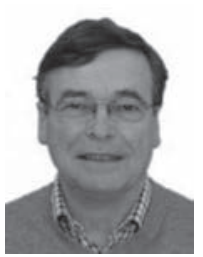

François Laudenbach [francois.laudenbach @univ-nantes.fr] is a topologist (exploring topics such as pseudo-isotopy, Morse functions, 2-spheres in 3-manifolds, closed differential forms of degree one, generating functions in symplectic topology and Morse-Novikov theory). He has had positions as professor at various places including the University Paris-sud (Orsay) from 1974 to 1989 and then successively the École Normale Supérieure de Lyon, the École polytechnique and the Université de Nantes, where he retired as a professor emeritus. He has been Editor-in-Chief of Astérisque and of the Bulletin SMF at different periods. He directed the Centre de Mathématiques of the École polytechnique from 1994 to 2000. 